\title{
Mindfulness in Holistic Management of Schizophrenia
}

\section{Pronab Ganguly ${ }^{\star}$}

Western Sydney University, Sydney, Australia

\section{Abstract}

Mindfulness based intervention (MBI) has been found to promote well-being by reducing symptoms of stress and non-reactivity to experience. It has been found to facilitate post-traumatic growth and enhance vigour while relieving fatigue. It can contribute effectively to apathy and self-awareness. Schizophrenia patients suffer from lack of motivation, apathy, boredom and social isolation to name a few. Holistic management of schizophrenia refers to management of quality of life for schizophrenia patients in its totality. This includes stable accommodation, healthy relationship, education, training, employment, social inclusion, integration with the community and entertainment etc. As mindfulness helps gain control over mind and regulate emotions, it can be helpful to alleviate some of the symptoms of schizophrenia patients. This study identifies the issues in holistic management of schizophrenia and investigates how mindfulness based intervention can contribute to them effectively. In the introduction section we have explored the implication and importance of holistic management of schizophrenia and features of mindfulness. In method section, we have investigated the various issues and symptoms of holistic management of schizophrenia and how mindfulness based intervention can address them. In discussion, we have reviewed research studies associated with mindfulness for schizophrenia patients and identified the scope of further work for mindfulness to be meaningful for schizophrenia patients.

\section{Introduction}

Schizophrenia is a mental disorder that impacts how a person acts, thinks, and perceives the world [1]. Schizophrenia is a chronic psychiatric disorder characterized by symptoms such as delusions, hallucinations, disorganised speech and behaviours, abolition and diminished emotional expression [2]. The cause of these symptoms has been attributed to dysregulation of dopaminergic signalling [3]. Schizophrenia is considered amongst the topmost 10 common disorders in the world [4]. About $1 \%$ of the general population suffers from schizophrenia [5]. The social and economic costs of treating and caring for schizophrenia patients far outweigh its occurrence. The long-term disability burden related to schizophrenia is far greater compared to any other mental disorder [6]. The direct cost of schizophrenia amounts to $1.4-2.8 \%$ of national health care budget and is almost up to $20 \%$ of the direct expenses of all types of mental health costs in most of the developed nations [7]. The indirect costs such as independent accommodation, financial support, supported employment and training are comparable to the direct costs and may be even more. Importantly, the aim of treating this disorders is not only decreasing some of the symptoms, but also enhance quality of life and engage patients in everyday life (by having successful jobs, relationships among others).Though primarily it involves pharmacological intervention, holistic management also offers the management of issues related to the symptoms of schizophrenia by providing suitable and secure accommodation, education and training, diet management, counselling, yoga, tai-chi and independent living skill amongst others. In pharmacological intervention, antipsychotic drugs are administered in various combinations in a patient specific manner. Patients respond to the combination drugs in their own unique way. Mainstream pharmacological treatment reduces the positive symptoms of schizophrenia to a reasonable extent but residual symptoms still persist. Cognitive behaviour therapy (CBT) is helpful in reducing the negative symptoms as well as improving the daily quality of life. CBT is widely used in United Kingdom and is recommended in USA along with mainstream pharmacological intervention. It encourages the patients to go for education, job and

\section{Publication History:}

Received: September 20, 2018

Accepted: November 26, 2018

Published: November 28, 2018

\section{Keywords:}

Mindfulness, Schizophrenia, Holistic, Management training. Yoga and tai chi contribute to overall sense of well-being. Although most pharmacological and counselling techniques focus on reducing positive and negative symptoms, other issues such as homelessness, lack of education, training, employment, independent living skill and recreation equally impact the quality of life in individuals with schizophrenia. Thus, targeting these symptoms is of paramount importance. Suitable and secure accommodation is highly importantas homelessness is detrimental to the pharmacological intervention. Similarly the provision of employment through suitable education and training helps the patients to integrate with the community. Since a major portion of the patients are not employed and on government income support system, they lead a poor quality of life and, thus, get attracted to smoking and other substance abuse. Schizophrenia patients are lonely and bored. They need friendship and healthy relationship with the family and friends. Thus, if all the issues related to quality of life are provided, the schizophrenia patients can lead a fulfilling and meaningful life. Basically the loss of quality of life for schizophrenia patients is due to deficient thoughts and feelings. This deficiency arises from negative thoughts, lack of self- awareness, emotional pain and asociability among others. Mindfulness based intervention can address some of these issues. Hence a framework of mindfulness can contribute to holistic management of schizophrenia in a significant manner.

Mindfulness-based intervention (MBI) is supposed to assist patients with schizophrenia respond differently to their psychotic symptoms by readjusting their awareness and judgement free acceptance of feelings and thoughts. It prepares a more suitable strategy of coping and control over those psychotic experiences.

"Corresponding Author: Mr. Pronab Ganguly, Western Sydney University, Sydney, Australia; E-mail: pronabganguly@gmail.com

Citation: Ganguly P (2018) Mindfulness in Holistic Management of Schizophrenia Int J Psychol Behav Anal 4: 156. doi: https://doi.org/10.15344/2455-3867/2018/156

Copyright: (c) 2018 Ganguly. This is an open-access article distributed under the terms of the Creative Commons Attribution License, which permits unrestricted use, distribution, and reproduction in any medium, provided the original author and source are credited. 
It has been reported [8] that MBI could provide various types of benefits for patients with schizophrenia. MBI assists the patients to regain control without the negative side effects. MBI is considered to strengthen the capability to adjust and tolerate negative thoughts and feelings for the schizophrenia patient and attain a sense of calmness and peace of mind. MBI is very effective to strengthen self-regulation and management of the disorder. Most of the other psychosocial interventions provide limited techniques for motivation of the patients into self-management of the disorder. They seldom help the patients develop an acceptance of the disorder. MBI is quite effective in this respect.

Mindfulness may be considered as awareness as well as focused attention to momentary experiences and feelings with a nonjudgmental attitude. It provides exploration of inner experience with calmness and no reaction. By MBI a schizophrenia patient is ready to accept dysfunctional thoughts and feelings non-judgmentally. The patient can get rid of impaired cognitive, emotional and behavioural symptoms. This way MBI can shape patient's emotion and thought process in a more constructive pattern. The long-term effects of MBI include improvement in psychotic functioning, insight into the disorder and relapse prevention. Mindfulness consists of two cognitive processes such as self-regulation of attention and an attitude of curiosity, openness, and acceptance for all aspects of the momentary experience, including thoughts, feelings, and sensations. $\mathrm{MBI}$ is closely linked to well-developed self-regulation by impacting on attentional control, emotion regulation, and self-awareness. At the beginning, there was some hesitation about MBI application for schizophrenia patients as MBI is generally linked to intensive meditation practice that may impact psychosis and mania. However, the type of MBI applied to schizophrenia patients is short in nature and is typically brief (15-45 minutes). This type of MBI is varied from intensive type that includes fasting and sleep deprivation. In research studies [8] meditation for 10 minutes has been suggested for schizophrenia patients.

\section{Method}

Research studies indicate that MBI can reduce rehospitalization frequency, improve features of neurocognition and contribute positively to clinical outcome in schizophrenia patients. In early psychosis, MBI is supposed to improve emotion regulation, anxiety, and depression. Furthermore a couple of studies have now indicated that MBI may improve negative symptoms in schizophrenia. Actually a meta-analysis suggested that, while MBI are moderately effective in improving several aspects of psychotic disorders, the effects on negative symptoms are higher than for positive symptoms. Although these preliminary indications are encouraging we still do not have effective therapy for persistent and debilitating negative symptoms, such as avolition, anhedonia etc.

MBI can contribute effectively to negative thoughts, less motivation in life, feelings of lack of interest, lack of interest in people's conversations, lack of self-awareness, anhedonia, behavioural inhibition, dysfunctional attitudes, and rejection of life, boredom and asociality for schizophrenia patients as summarised in table 1 . A lower behavioural inhibition normally leads to a lesser willingness to be away from non-conducive stimulations. Mindfulness develops a willingness to accept all aspects of immediate experience [9]. Thus mindfulness can be considered as a therapy to observe and tolerate unpleasant internal sensations, without responding to avoidance $[10,11]$.Schizophrenia patients generally suffer from high levels of negative effects and utilise less effective mechanisms for regulating emotions [12,13]. Mindfulness can lead to improved regulation of emotion [14]. MBI may help to address dysfunctional attitudes in schizophrenia. MBI may prevent relapse in depression [15]. Mindfulness allows schizophrenia patients to view negative, depressing thoughts as passing events that do not reflect the real life scenarios [16]. This detached view reduces the strength that negative beliefs have on the behaviour of schizophrenia patients. Lack of interest in life is an issue with schizophrenia patients. Patients generally adopt strategies such as distraction, avoidance and suppression [17]. This avoidance strategy produces distress and anxiety, which are closely linked with the risk of relapse and re-hospitalization [18]. As mindfulness based intervention improves the awareness of self and others, it can help in this respect. Some of the schizophrenia patients sometimes loose themselves in the rumination and symptoms [17]. As MBI concentrates on focus, it can help in that respect also. MBI may positively contribute to self-awareness and might be helpful to relate to other people's conversation. Mindfulness can deployed for reaction to negative thoughts more quickly as a function of improved attention and heightened regulatory control. Mindfulness is found to support acting with awareness that was associated with lower behavioural inhibition. Non-judging is related to lower behavioural inhibition and non-reacting was associated with higher behavioural activation [8]. Mindfulness promotes these kinds of behaviour. Anhedonia refers to inability to feel pleasure in normally pleasurable activities. In schizophrenia, anhedonia reflects abnormal psychological processes, such as low-pleasure beliefs and reduced estimation of past and future pleasure, as well as dysfunctional behavioural aspects such as decreased pleasure-seeking behaviour. As mindfulness promotes non-judgemental mentality, it contributes favourably to these kinds of behaviour. Behavioural inhibition is sensitive to negative stimulation and activated by anxiety and innate fear stimuli and is responsible for ceasing or inhibiting behaviour. Some facets of mindfulness were also associated favourably with behavioural activation and inhibition [8]. Dysfunctional attitudes have been found to mediate the relationships between neurocognition and negative symptoms and functioning. MBI may contribute positively in improvement of dysfunctional attitude. Avolition refers to decrease in the motivation to initiate and perform self-directed purposeful activities. For schizophrenia patients, avolition indicates that the patient is seeking to complete a task but is unable to gather the mental and physical energies to do so. Mindfulness based training may be effective in addressing this issue. If a schizophrenia patient is suffering from auditory or visual delusions and paranoia, they seldom engage in day-to-day activities because the voices and suspiciousness are directing their activities. MBI may be able to regulate emotions and reduce the effect of psychosis. Apathy has significant functional results on the patient's quality of life. Apathy has direct effect on the social and professional life of a schizophrenia patient. Thus schizophrenia patients loose autonomy, employment and social integrationdue to apathy [19]. As MBI contributes to regulation of emotion, it may help to reduce apathy. In schizophrenia, boredom is associated with a host of undesirable outcomes. Boredom generates psychotic mood disturbances, increased substance-abusing behaviours, paranoia and hallucinations, changes in distractibility and overall cognitive inefficiency. MBI based boredom coping skills may be integrated into the social skills training and rehabilitation strategies for schizophrenia patients. Various issues in holistic management of schizophrenia and associated MBIs are summarised in table 1.

\section{Discussion}

In this research study [8] mindfulness in schizophrenia was investigated. They hypothesized that schizophrenia patients would

Int J Psychol Behav Anal

ISSN: 2456-3501

IJPBA, an open access journal Volume 4. 2018. 156 
Citation: Ganguly P (2018) Mindfulness in Holistic Management of Schizophrenia. Int J Psychol Behav Anal 4: 155. doi: https://oi.org/10.15344/2455$3867 / 2018 / 155$

Page 3 of 4

\begin{tabular}{|c|c|c|}
\hline $\begin{array}{l}\text { Issues in holistic management of } \\
\text { schizophrenia }\end{array}$ & Possible effect of mindfulness & Related studies \\
\hline Emotional regulation & $\begin{array}{l}\text { Mindfulness reduces depressive symptoms through greater } \\
\text { emotion regulation. }\end{array}$ & {$[12-14]$} \\
\hline Less motivation in life & $\begin{array}{l}\text { Mindfulness may improve motivation by reduction of negative } \\
\text { thoughts. }\end{array}$ & {$[9-11]$} \\
\hline Feelings of lack of interest in others & Mindfulness enhances social skill. & {$[20]$} \\
\hline $\begin{array}{l}\text { Lack of interest in people's } \\
\text { conversations }\end{array}$ & Mindfulness helps to improve relationship. & {$[20]$} \\
\hline Negative thoughts & $\begin{array}{l}\text { Mindfulness helps to assess thoughts with detachment, thereby, } \\
\text { lessening the impact of negative thoughts. }\end{array}$ & {$[16]$} \\
\hline Lack of self-awareness & $\begin{array}{l}\text { Mindfulness contributes to increased clarity in thinking and } \\
\text { perception. }\end{array}$ & {$[8]$} \\
\hline Anhedonia & $\begin{array}{l}\text { Mindfulness improves focus and reduces negative emotional } \\
\text { reaction for pleasurable activities. }\end{array}$ & {$[21]$} \\
\hline Behavioural inhibition & Mindfulness enhances experience of feeling connected. & {$[8,21]$} \\
\hline Dysfunctional attitudes & Mindfulness reduces fixation on negative emotions. & {$[22]$} \\
\hline Avolition, & $\begin{array}{l}\text { Mindfulness initiates a change in mind, thereby, giving it a } \\
\text { purpose. }\end{array}$ & [23] \\
\hline Rejection of life & $\begin{array}{l}\text { Mindfulness enhances experience of being calm and internally } \\
\text { still, thereby, embracing life. }\end{array}$ & \\
\hline Boredom & Mindfulness inspires to enjoy life, thereby, reducing boredom. & {$[24]$} \\
\hline Asociality & $\begin{array}{l}\text { Mindfulness reduces being argumentative and defensive with } \\
\text { friends and family, thereby, enhancing sociability. }\end{array}$ & {$[25]$} \\
\hline Apathy & Mindfulness gives a direction for life reducing apathy. & [19] \\
\hline
\end{tabular}

Table 1: Issues in holistic management of schizophrenia and possible effects of mindfulness.

have less mindfulness than controls. Secondly they investigated relations between mindfulness and assessments of negative symptoms in patients. It was hypothesised that higher mindfulness would relate to lower negative symptoms. Thirdly, it was studied how mindfulness was related to negative symptoms and adaptive functioning in schizophrenia. They hypothesized that mindfulness would be related lower behavioural inhibition, greater behavioural activation, more adaptive emotion regulation in schizophrenia patients. Finally, investigative analysis was carried out to identify the relationship between mindfulness to positive symptoms and cognition. It was identified that schizophrenia patients showed reduced levels of overall mindfulness than control participants. They did not find any evidence that greater mindfulness would be inversely related with negative symptoms. It also could not be found that any significant relationships exist between mindfulness and positive symptoms. Their research study although indicated evidence that there are three ways in which mindfulness support more productive and quality of life for patients with schizophrenia. It was found that mindfulness might reduce behavioural inhibition, increase adaptive emotion regulation, and provide detachment from dysfunctional attitudes.

In another research study [26] the applicability of meditation to schizophrenia patients' persistent negative symptoms was studied. Meditations showed promise for reducing negative symptoms such as anhedonia, avolition, and asociality while increasing the impact of factors for psychiatric recovery such as hope and purpose in life. Attempts were made to identify how to conduct mindfulness with patients with negative symptoms, the potential benefits to the patients, and problem that may arise. Meditation showed to be a promising intervention as there are very few treatments options available for patients with negative symptoms.
In a third study [27] MBI for Chinese people with schizophrenia was investigated. A trial was conducted with 107 patients with schizophrenia: 36 received a 6-month MBI intervention programme and 35 received conventional psychoeducation programme. The outcomes measured were psychiatric symptom severity, social functioning, social support, insight into illness and frequency and duration of rehospitalization. The MBI intervention group reported higher improvements in psychiatric symptoms, psychosocial functioning, insight into illness/treatment and duration of rehospitalization over 24 months when compared with the other group. So it was reported that MBI seems to be a good approach to treatment for Chinese patients with schizophrenia.

In a fourth study [28] the effects of MBI was tested in a group program. In that study for schizophrenia patients, MBI along with usual care was compared with conventional psycho-education program as well as treatment-as-usual over a 6-month follow-up period. About 69 outpatients with schizophrenia were randomly divided into the three groups in this study. MBI patients showed greater reductions in their psychotic symptoms and duration of rehospitalization over 6-month follow-up period. Patients in MBI group also showed greater improvements in their insight into illness and level of functioning than the other two groups. The study concluded that for schizophrenia patients MBI can contribute positively not only in terms of their mental state and risk of relapse but also their insight into illness and psychosocial functioning.

Schizophrenia is generally treated with various antipsychotic drugs as well as therapies. The drugs sometimes are not effective and may have some undesirable side effects. Hence, there is a need for safe and effective complimentary therapy for schizophrenia patients. MBI 
is considered to be helpful for a variety of mental health problems, including anxiety, depression, impulsivity, phobias, post-traumatic stress disorder, sexual dysfunction, and suicidal ideation. It seems to be helpful with psychosis as well. Hence, there is a need to further investigate the potential benefits of mindfulness as a therapy for schizophrenia. Hence it is required to study the relationships of MBI to the positive and negative symptoms of schizophrenia in further depth. Thus we find that there are potential benefits of mindfulness based intervention in holistic management of schizophrenia. More thorough and systematic research and clinical studies are required to investigate this issue further.

\section{Competing Interests}

The author declare no competing interests.

\section{References}

1. Coyle JT (2017) Schizophrenia: Basic and Clinical. Adv Neurobiol 15: 255280.

2. Shenton ME, Kikinis R, Jolesz FA, Pollak SD, LeMay M (1992) Abnormalities of the Left Temporal Lobe and Thought Disorder in Schizophrenia-A Quantitative Magnetic Resonance Imaging Study. N Engl J Med 327: 604612.

3. American Psychiatric Association (2013) Diagnostic and statistical manual of mental disorders. Arlington, VA: American Psychiatric Publishing.

4. Mathers CD, Loncar D (2006) Projections of global mortality and burden of disease From 2002 to 2030.PLoS Medicine 3: e442.

5. Simeone JC, Ward AJ, Rotella P, Collins J, Windisch R, et al. (2015) An evaluation of variation in published estimates of schizophrenia prevalence from 1990 - 2013: A systematic literature review. BMC Psychiatry 15: 193.

6. Neil AL, Carr VJ, Mihalopoulos C, Mackinnon A, Lewin TJ, et al. (2014) What difference a decade? The costs of psychosis in Australia in 2000 and 2010 : Comparative results from the first and second Australian national surveys of psychosis. Aust N Z J Psychiatry 48: 237-248.

7. Galletly C, Castle D, Dark F, Humberstone V, Jablensky A, et al. ( 2016) Royal Australian and New Zealand College of Psychiatrists clinical practice guidelines for the management of schizophrenia and related disorders. Aust N Z J Psychiatry 50: 410-472.

8. Tabak NT, Horan WP, Green MF (2015) Mindfulness in schizophrenia: Associations with self-reported motivation, emotion regulation, dysfunctional attitudes, and negative symptoms. Schizophr Res 168: 537542.

9. Bishop SR, Lau M, Shapiro S, Carlson L, Anderson ND, et al. (2004) Mindfulness: a proposed operational definition. Clin Psychol Sci Pract 11: 230-241.

10. Baer RA (2003) Mindfulness training as a clinical intervention: a conceptual and empirical review. Clin Psychol Sci Pract 10: 125-143.

11. Treanor $M$ (2011) The potential impact of mindfulness on exposure and extinction learning in anxiety disorders. Clin Psychol Rev 31: 617-625.

12. Horan WP, Blanchard JJ, Clark LA, Green MF (2008) Affective traits in schizophrenia and schizotypy. Schizophr. Bull 34: 856-874

13. Kimhy D, Vakhrusheva J, Jobson-Ahmed L, Tarrier N, Malaspina D, et al. (2012) Emotion awareness and regulation in individuals with schizophrenia: implications for social functioning. Psychiatry Res 200: 193-201.

14. Tang YY, Holzel BK, Posner MI (2015) The neuroscience of mindfulness meditation. Nat Rev Neurosci 16: 213-225.

15. Piet J, Hougaard E (2011) The effect of mindfulness-based cognitive therapy for prevention of relapse in recurrent major depressive disorder: a systematic review and meta analysis. Clin Psychol Rev 31: 1032-1040.

16. Teasdale JD, Segal ZV, Williams JMG, Ridgeway Va, Soulsby JM, et al. (2000) Prevention of relapse/recurrence inmajor depression by mindfulness-based cognitive therapy. J Consult Clin Psychol 68: 615-623.

17. Jacobsen P, Morris E, Johns L, Hodkinson K (2011) Mindfulness groups for psychosis; key issues for implementation on an inpatientunit. Behav Cogn Psychother 39: 349-353.

Int J Psychol Behav Anal

ISSN: 2456-3501
18. van der Valk R, van de Waerdt S, Meijer CJ, van den Hout I, de Haan L, et al. (2013) Feasibility of mindfulness-based therapy in patients recovering from a first psychotic episode: a pilot study. Early Interv Psychiatry 7: 64-70.

19. Yazbek H, Raffard S, Del-Monte J, Pupier F, Larue A, et al. (2014) The clinic of apathy in schizophrenia: a critical review of the issue. Encephale 40: 231239.

20. Neto D (2018) The Place of Meditation in the Health Care System. Int J Psychol Behav Anal 4: 147.

21. Reddy LF, Green MF, Rizzo S, Sugar CA, Blanchard J, et al. ( 2014) Behavioral Approach and Avoidance in Schizophrenia: An Evaluation of Motivational Profiles. Schizophr Res 159: 164-170.

22. Quinlan T, Roesch S, Granholm E (2014) The role of dysfunctional attitudes in models of negative symptoms an Chien WT, Lee IY (2013) The mindfulnessbased psychoeducation program for Chinese patients with schizophrenia. Psychiatr Serv 64: 376-379.d functioning in schizophrenia. Schizophr Res 157: 182-189.

23. Foussias G, Remington G (2010) Negative Symptoms in Schizophrenia: Avolition and Occam's razor. Schizophr Bull 36: 359-369.

24. Todman M (2003) Boredom and psychotic disorders: cognitive and motivational issues. Psychiatry 66: 146-167

25. Grant PM, Beck AT (2010) Asocial beliefs as predictors of a social behavior in schizophrenia. Psychiatry Res 177: 65-70.

26. Lam AHY, Chien WT (2016) The effectiveness of mindfulness-based intervention for people with schizophrenia: a systematic review. Neuropsychiatry 6: 208-222.

27. Chien WT, Lee IY (2013) The mindfulness-based psychoeducation program for Chinese patients with schizophrenia. Psychiatr Serv 64: 376-379.

28. Wang LQ, Chien WT, Yip LK, Karatzias T (2016) A randomized controlled trial of a mindfulness-based intervention program for people with schizophrenia: 6-month follow-up. Neuropsychiatr Dis Treat 12: 3097-3110. 